\title{
Advance Malignant Phyllodes Tumor with Active Bleeding in Children: A Rare Case
}

\author{
Dedy Hermansyah ${ }^{1 *}$, Fernando Silalahi ${ }^{2}$, Albiner Simarmata ${ }^{2}$, Denny Rifsal Siregar ${ }^{1}$ \\ ${ }^{1}$ Division of Surgical Oncology, Department of General Surgery, Universitas Sumatera Utara, Medan, Indonesia \\ ${ }^{2}$ Division of Surgical Oncology, Department of General Surgery, Dr. Pringadi General Hospital, Medan, Indonesia
}

\section{ARTICLE INFO}

Received : 05 November 2020

Reviewed : 19 February 2021

Accepted : 21 March 2021

Keywords:

breast, children, phyllodes tumor

*Corresponding author:

Dedy Hermansyah

Division of Surgical Oncology,

Department of General Surgery,

Universitas Sumatera Utara, Medan,

Indonesia

dedi.hermansyah@usu.ac.id

\begin{abstract}
Introduction: Phyllodes tumors are fibroepithelial breast lesions that are uncommon in women and rare among children. Due to scarcity, there are only a few large pediatric phyllodes tumor series. Current guidelines do not differentiate treatment recommendations between children and adults.
\end{abstract}

Case Presentation: A 12-year-old girl presented with a lump in her right breast which grew rapidly within a month. On physical examination, we found a mass on the right breast sized \pm 10 $x 10 \mathrm{~cm}$; the lump was fragile and bled easily. The patient's condition was getting worse and worse. Due to this deterioration, we performed a mastectomy immediately.

Conclusions: The incidence of malignant phyllodes tumor in children is very rare and required prompt treatment in emergency cases.

\section{INTRODUCTION}

Phyllodes tumor peak incidence in women is between the age of 35 to 55 years. The incidence rate of all breast malignancies is < $1 \%$ in approximately 2.1 per million population. Most of these tumors are benign, but some have the potential to become malignant [1].

Phyllodes tumor lesions are often unilateral, single, nodular, have a painless mass with insidious onset, and slow progression. Most patients have a short-term history of rapid mass growth. The tumor rapidly grows and can push against the chest wall. Tumor volume varies widely as several studies have reported tumors ranging in size from less than $1 \mathrm{~cm}$ to a maximum diameter of $40 \mathrm{~cm}$. The relationship between tumor size and prognosis remains unclear. These tumors grow radially and compress the surrounding breast parenchyma, where a false capsule is created through which the tumor extends and grows into the rest of the healthy mammary tissue [2-4].

Malignant phyllodes tumor exhibits a stroma characterized by cellularity and atypia, has a permeative margin, and has a mitotic activity of at least 10/10 field area. Stromal overgrowth is usually easy to identify [2].

Breast lumps are rare in children. The most common breast lump found in the adolescent population is fibroadenoma. Phyllodes tumor is a rare fibroepithelial tumor that accounts for $0.3-0.5 \%$ of all breast tumors in women. They are rarely observed in adolescents with only 20 cases reported [5-7]. Here, we report the case of a teenager with a lump in her right breast which, according to the histopathology, was identified as a malignant phyllodes tumor.

\section{CASE PRESENTATION}

A 12-year-old girl presented with a complaint of a lump in the right breast for the last 3 months. The lump was growing rapidly in the last 1 month. The patient previously underwent surgical removal of the tumor on the right breast 1 year ago. She had a history of menarche at the age of 11 years, was not married, and had no history of suffering from the same disease in the family.

At the beginning of her visit, the patient came in a good general condition and underwent several initial examinations (screening) such as routine blood tests and chest X-rays. However, a week later, she came with symptoms of deterioration of her general condition, in which her $\mathrm{Hb}$ decreased to $4 \mathrm{~g} / \mathrm{dL}$ from the initial $\mathrm{Hb}$ of $10.8 \mathrm{~g} / \mathrm{dL}$.

On physical examination, there was a lump on the right breast measuring $\pm 10 \times 10 \mathrm{~cm}$. The lump was 
fragile and bled easily. There was no enlargement of lymph nodes in the right axilla, and the left breast was within normal limits. Since the patient had deteriorated general condition due to active bleeding from the tumor mass, we decided to perform mastectomy immediately.

The patient underwent simple mastectomy surgery with bleeding during an operation of about $\pm 50 \mathrm{cc}$ before being treated for 3 days with daily drain production of $\pm 20 \mathrm{cc}$. The pre and operative finding are shown in Figure 1. Histopathological analysis of the Haematoxylin and Eosin-stained specimens showed a biphasic tumor and enlarged histopathological features showing features of nuclear pleomorphism and mitosis
(Figure 2). She underwent treatment at the polyclinic for \pm 2 weeks with good/dry surgical wound results, and the drain was removed after 5 days. After the surgical management, we also performed external radiation as adjuvant therapy. The 7 months follow-up result can be seen in Figure 3.

\section{DISCUSSION}

Evaluations in children presenting with a lump in the breast differ substantially from the evaluations in adults because of the marked differences in breast cancer risk and breast architecture. There is little
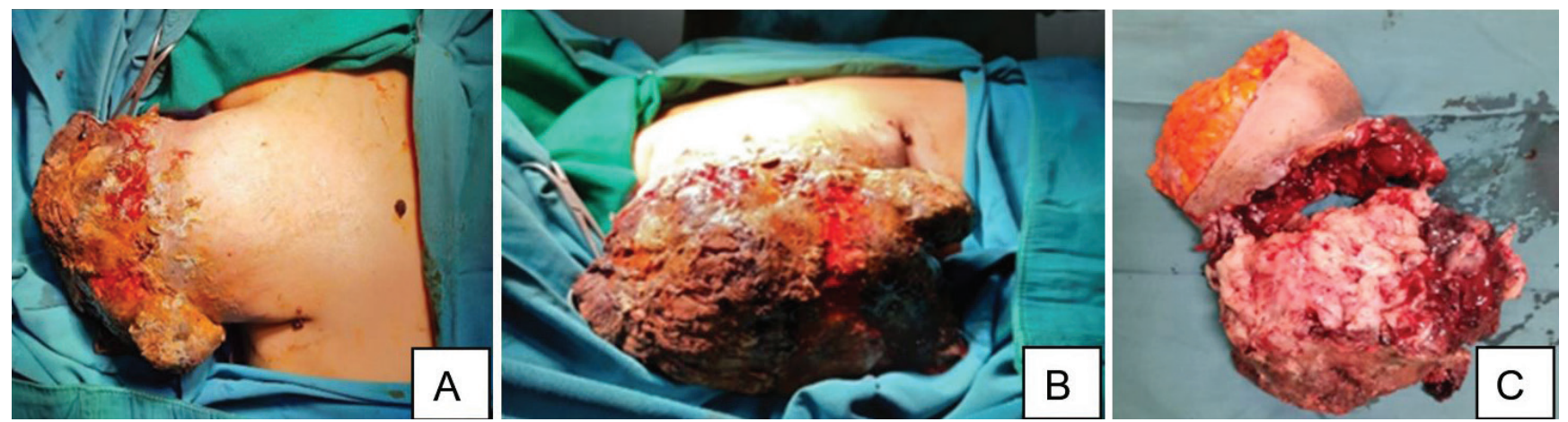

Figure 1. (A) Malignant pre-op condition (R) phyllodes; (B) Malignant phyllodes pre-op (R) conditions;

(C) Operative findings of malignant phyllodes

Figure 2. Histopathological analysis of the Haematoxylin and Eosin-stained specimens
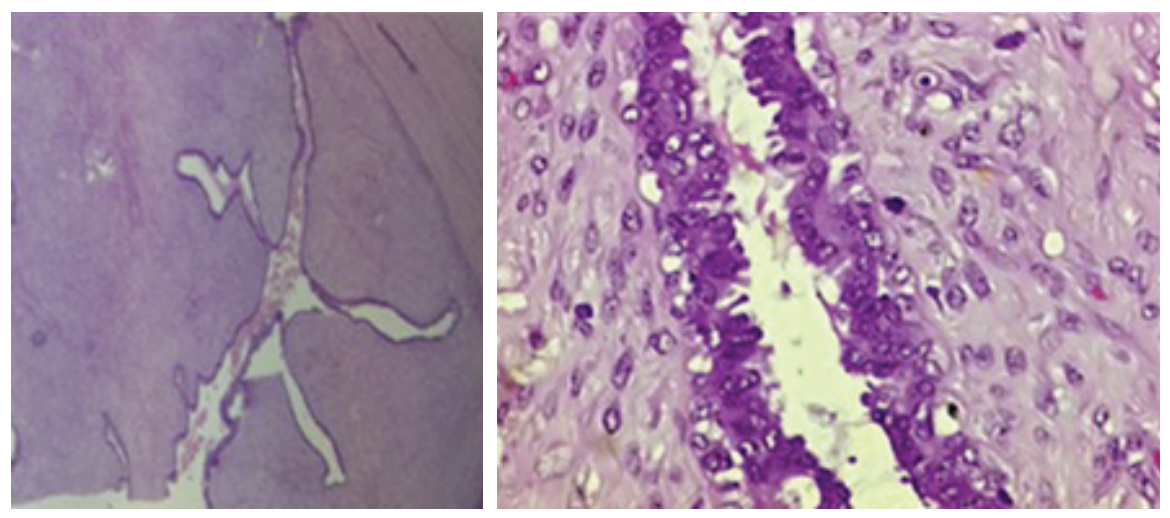

Figure 3. Clinical photos after 7 months follow up
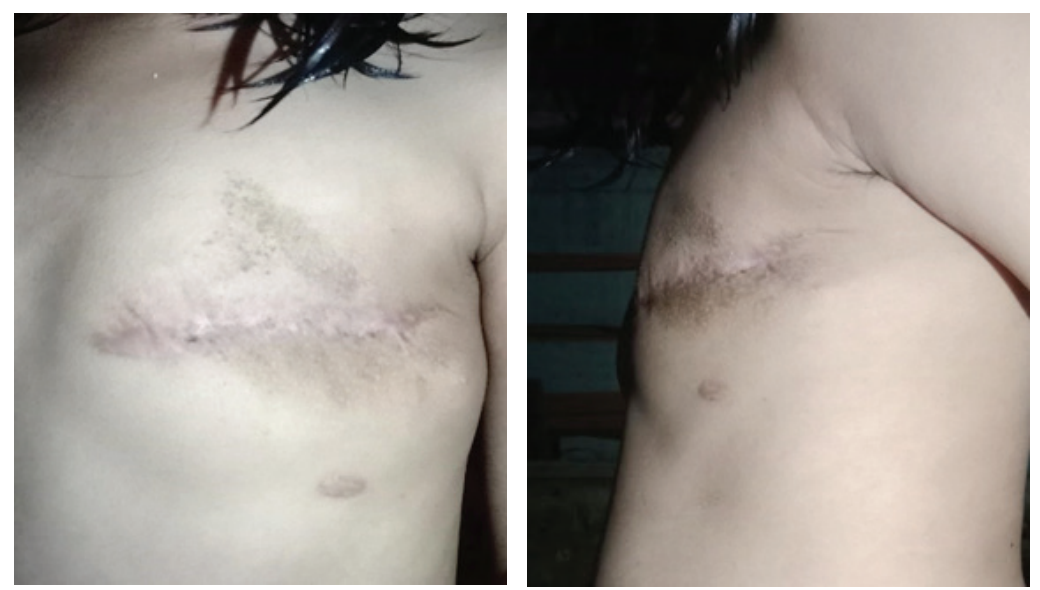
emphasis on the exclusion of malignancy since breast lumps in children are usually benign ( $95 \%$ benign fibroadenoma) [1,2].

Due to the lack of tumor-free margins during the surgical resection, a recurrence rate of less than $13 \%$ is usually observed. A close follow-up with frequent breast examinations and imaging tests is recommended after surgery. A breast lump with a history of rapid growth and an ultrasound image showing a fibroadenoma (unless the size is $>2 \mathrm{~cm}$ ) should raise high suspicion for a phyllodes tumor. Axillary node involvement is rare. Another characteristic feature of this tumor is a high local recurrence (5-20\%). The most common benign variant is only $10-25 \%$ of malignant cases. The rate of distant metastases for malignant tumors is $15-25 \%$ [1-3].

The only treatment option for this tumor is surgical removal. After the diagnosis, the phyllodes tumors are treated with a wide excision. If it is large, a mastectomy may be necessary. A recent study by Yom et al. concluded that a clear margin of $0.1 \mathrm{~mm}$ was equivalent to a margin of $1 \mathrm{~cm}[5,7]$.

Incisional and excisional biopsy is the definitive method for diagnosing phyllodes tumors. Complete surgical resection is the treatment of choice; however, particularly in frontier and malignant phyllodes tumors, the rate of resection is controversial because it penetrates the surrounding healthy tissue. Therefore, wide local excision is performed covering the healthy breast tissue. No proven curative or palliative role in tumor management is confirmed for neoadjuvant and adjuvant therapy because of the lack of tumor-free margins during surgical resection. The recurrence rate is usually less than $13 \%[3,5,8]$.

Total mastectomy or breast-conserving surgery was the gold standard of treatment for this case, and adjuvant postoperative radiotherapy has been shown to reduce local recurrence rates. Revision surgery may be required for most tumors with inadequate margin removal, and radiotherapy after breast surgery can significantly reduce local recurrence rates for borderline and malignant tumors $[1,10]$.

A close follow-up with breast examination and imaging tests is recommended after surgery. Five-year survival rates were found in nearly $100 \%$ of patients with benign types, $98 \%$ with borderline types, and about $88 \%$ with malignant types $[1,9,10]$.

\section{CONCLUSIONS}

The incidence of malignant phyllodes tumor in children is very rare and requires prompt treatment in emergency cases. In principle, the handling of phyllodes tumor cases in children is the same as in adults.

\section{DECLARATIONS}

\section{Ethics of Approval Consent to Participate NA}

\section{Competing of Interest}

The authors declare no competing interest in this study.

\section{Acknowledgment}

The authors wish to thank Universitas Sumatera Utara General Hospital and all parties who contributed to this research.

\section{REFERENCES}

1. Zhou ZR, Wang CC, Yang ZZ, et al. Phyllodes tumors of the breast: diagnosis, treatment and prognostic factors related to recurrence. J Thorac Dis. 2016;8(11):3361-8.

2. HHS Public Access. Phyllodes tumours of the breast: a consensus review. Histopathology. 2016;68(1):5-21.

3. Makhija D, Shah H, Bothra J, Jayaswal S. An adolescent with a phyllodes tumor: A case report and review. Int J Pediatr Adolesc Med. 2016;3(4):180-3.

4. Erginel B, Celet Ozden B, Yesil Onder S, et al. Management of a benign phyllodes tumor in a 13-year-old girl with trans-position of the nipple areola complex and breast reconstruction. Acta Chir Belg. 2015;115(3):256-9.

5. Pruthi S, Jones KN, Boughey JC, Simmons PS. Breast masses in adolescents: clinical pearls in the diagnostic evaluation. Am Fam Physician. 2012;86(4):325-6.

6. Wang $\mathrm{H}$, Wang $\mathrm{X}$, Wang CF. Comparison of clinical characteristics between benign borderline and malignant phyllodes tumors of the breast. Asian Pac J Cancer Prev. 2014;15(24):10791-5.

7. Chu JS. Prognostic factors in phyllodes tumor of the breast: are immunohistological biomarkers useful?. J Chin Med Assoc. 2004;67(1):1-2.

8. Liew KW, Siti Zubaidah S, Doreen L. Malignant phyllodes tumors of the breast: A single institution experience. Med J Malaysia. 2018;73(5):297-300.

9. Yohe S, Yeh IT. "Missed" diagnosis of Phyllodes tumor on breast biopsy: pathological clues to its recognition. Int J Surg Pathol. 2008;16(2):137-42.

10. Farias-Eisner GT, Small K, Swistel A, Ozerdem U, Talmor M. Immediate implant breast reconstruction with acellular dermal matrix for treatment of a large recurrent malignant phyllodes tumor. Aesthetic Plast Surg. 2014;38(2):373-8. 